Soc. Colloq. Publ. Vol. 31, Amer. Math. Soc., Providence, R. I., rev. ed., 1957.

7. N. Jacobson, Lie algebras, Interscience, New York, 1962.

8. E. Nelson, Analytic vectors, Ann. of Math. 70 (1959), 572-615.

9. L. Pontrjagin, Topological groups, Princeton Univ. Press, Princeton, N. J., 1939.

10. I. E. Segal, $A$ class of operator algebras which are determined by groups, Duke Math. J. 18 (1951), 221-265.

11. S. Yosida, On the differentiability and the representation of one parameter semigroup of linear operators, J. Math. Soc. Japan 1 (1948), 15-21.

PRINCETON UNIVERSITY AND

University of California, Berkeley

\title{
WEAK LEVI CONDITIONS IN SEVERAL COMPLEX VARIABLES ${ }^{1}$
}

\author{
BY AVNER FRIEDMAN
}

Communicated by R. P. Boas, June 28, 1965

1. Introduction. Let $\Omega=\left\{z ; z \in \Omega_{0}, \rho(z)<0\right\}$ be a bounded domain in $C^{n}$, where $\rho \in C^{2}\left(\Omega_{0}\right), \Omega_{0}$ a neighborhood of $\Omega$, and let grad $\rho \neq 0$ on $\partial \Omega$. As is well known, if $\Omega$ is a domain of holomorphy then for any $x^{0} \in \partial \Omega$,

(1) $L\left(\rho\left(x^{0}\right), w\right) \equiv \sum_{j, k=1}^{n} \frac{\partial^{2} \rho\left(x^{0}\right)}{\partial z_{j} \partial \bar{z}_{k}} w_{j} \bar{w}_{k} \geqq 0 \quad$ whenever $\sum_{j=1}^{n} \frac{\partial \rho\left(x^{0}\right)}{\partial z_{j}} w_{j}=0$,

and, if (1) holds with strict inequality (for $w \neq 0$ ) then $\Omega$ is a domain of holomorphy. (1) is called the Levi condition (LC) and, in case of strict inequality, the strict LC. One of the consequences of the present work is that the above statement remains true if the assumption $\rho \in C^{2}$ is replaced by $\rho \in H^{2, \infty}$ (see $\$ 2$ ).

In what follows $\Omega$ is always given by $\rho$ as above, where $\rho \in C^{1}\left(\Omega_{0}\right)$, $\operatorname{grad} \rho \neq 0$ on $\partial \Omega$.

2. Definitions. If $\rho$ has second weak derivatives which belong to $L^{p}\left(\Omega_{0}\right)(1<p<\infty)$ then we say that $\Omega$ and $\rho$ belong to $H^{2, p}$. Actually we shall only need the derivatives $\partial^{2} \rho / \partial z_{j} \partial \bar{z}_{k}$ to belong to $L^{p}$, but then

1 This work was partially supported by the Alfred P. Sloan Foundation and by Nasa Grant NGR 14-007-021. 
necessarily $\partial^{2} \rho / \partial z_{j} \partial z_{k}$ are in $L^{p}$ in compact subsets. ${ }^{2}$ If the weak derivatives $\partial^{2} \rho / \partial z_{j} \partial \bar{z}_{k}$ are continuous, then we say that $\Omega$ and $\rho$ belong to $H^{2, \infty}$. The weak derivatives $\partial^{2} \rho / \partial z_{j} \partial z_{k}$, are not, in general, even in $L^{\infty} .8$

Let $t(z)$ be a $C^{\infty}$ function with support in $|z|<1, \int t(z) d \lambda=1$ $\left(d \lambda=\right.$ Lebesgue measure in $\left.C^{n}\right)$ and consider

$$
\rho_{\epsilon}(z)=\frac{1}{\epsilon^{2 n}} \int_{\Omega_{0}} \rho(\zeta) t\left(\frac{z-\zeta}{\epsilon}\right) d \lambda,
$$

for $\epsilon>0$ sufficiently small, in some neighborhood $\Omega_{*}$ of $\Omega\left(\bar{\Omega}_{*} \subset \Omega_{0}\right)$. If $\rho \in H^{2, p}(p<\infty)$ then, as $\epsilon \rightarrow 0, \rho_{\epsilon} \rightarrow \rho, D \rho_{\epsilon} \rightarrow D \rho$ uniformly in $\Omega_{*}$, $\int_{\Omega_{*}}\left|D^{2} \rho_{\mathrm{c}}-D^{2} \rho\right|^{p} d \lambda \rightarrow 0$; if $\rho \in H^{2, \infty}$ then the last relation is replaced by $\partial^{2} \rho_{\epsilon} / \partial z_{j} \partial \bar{z}_{k} \rightarrow \partial^{2} \rho / \partial z_{j} \partial \bar{z}_{k}$ uniformly in $\Omega_{*} ; D$ is any first order derivative.

Introduce positive functions $\phi_{p}(A), \psi_{p}(A)$ and $\eta(\epsilon)$ such that $\eta(\epsilon)$ $\rightarrow 0$ if $\epsilon \rightarrow 0$ and

$$
\begin{gathered}
\sum_{j, k}\left\{\int_{A}\left|\frac{\partial^{2}}{\partial z_{j} \partial \bar{z}_{k}}\left(\rho_{\epsilon}-\rho\right)\right|^{p} d \lambda\right\}^{1 / p} \leqq \phi_{p}(A) \eta(\epsilon), \\
\sum_{j, k}\left\{\int_{A}\left|\frac{\partial^{2} \rho}{\partial z_{j} \partial \bar{z}_{k}}\right|^{p} d \lambda\right\}^{1 / p} \leqq \psi_{p}(A) .
\end{gathered}
$$

If $\rho \in H^{2, p}(1<p \leqq \infty)$ then such functions clearly exist. We take

$$
\phi_{p}(A)=\psi_{p}(A)=C(\operatorname{vol} . A)^{1 / p} \text { if } \rho \in H^{2, \infty}(C=\text { const. } \neq 0) \text {. }
$$

3. Levi conditions.

Definition 1. Let $\Omega \in H^{2, p}$. We say that the weak Levi condition with index $p\left(\mathrm{WLC}_{p}\right)$ holds at $x^{0} \in \partial \Omega$ if for any $\epsilon>0$ there exist $\delta$ such that

$$
\begin{aligned}
& \int_{B_{\delta}} L(\rho(z), w(z)) d \lambda \\
& \quad \geqq-\epsilon\left\{\phi_{p}\left(V_{w}\right)+\psi_{p}\left(V_{w}\right)+\left(\operatorname{vol} . V_{w}\right)^{1 / p}\right\}\left\{\int_{B_{\delta}}|w(z)|^{2 q} d \lambda\right\}^{1 / q},
\end{aligned}
$$

for any $w \in C^{0}\left(B_{\delta}\right)$ satisfying

2 This follows from the inequality (z 1-dimensional) $\left({ }^{*}\right) \int_{A}|\partial w / \partial z|{ }^{p} d z \wedge d \bar{z} \leqq$ const. $\int_{B}\left(|\partial w / \partial \bar{z}|^{p}+|w|^{p}\right) d z \wedge d \bar{z}$ where $A$ is relatively compact to $B$. To prove $\left(^{*}\right)$ use integral representation for $w$ (which may be assumed to have compact support) in terms of $\partial w / \partial \bar{z}$ and $L^{p}$ estimates for singular integrals.

8 Indeed, in the contrary case, an argument involving the closed graph theorem yields an inequality of the form l.u.b. $A\left|\partial^{2} w / \partial z^{2}\right| \leqq$ const. l.u.b.B $\left(\left|\partial w / \partial z^{2} \partial z\right|+|\partial w / \partial z|\right.$ $+|\partial w / \partial \bar{z}|+|w|)$ (the same notation as in footnote 1) which is impossible (by [2]). 


$$
\sum_{j=1}^{n} \frac{\partial \rho(z)}{\partial z_{j}} w_{j}(z)=0 \quad \text { in } B_{\delta} .
$$

Here $V_{w}$ is the support of $w, 1 / q+1 / p=1, B_{\delta}=B\left(x^{0}, \delta\right)$ is the ball with center $x^{0}$ and radius $\delta$, and $\delta$ is sufficiently small such that $B_{\delta} \subset \Omega_{*}$ and grad $\rho \neq 0$ in $B_{\delta}$.

If we require $w$ to have weak derivatives in $L^{p}$, then all the results below remain unchanged; (5) then takes the equivalent form

$$
\begin{aligned}
\sum_{j, k} \int_{B_{j}} & \frac{\partial \rho}{\partial z_{j}} \frac{\partial w_{j}}{\partial \bar{z}_{k}} \bar{w}_{k} d \lambda \\
& \leqq \epsilon\left\{\phi_{p}\left(V_{w}\right)+\psi_{p}\left(V_{w}\right)+\left(\text { vol. } V_{w}\right)^{1 / p}\right\}\left\{\int_{B_{\delta}}|w| 2 q d \lambda\right\}^{1 / q} .
\end{aligned}
$$

Note however that if $\rho$ is not assumed to have second weak derivatives in $L^{p}$ then there may not exist any $w$ with first derivatives in $L^{p}$ which satisfies (6).

Definition 2. Let $\Omega \in H^{2, p}$. We say that the $\mathrm{WLC}_{p} I$ holds at $x^{0} \in \partial \Omega$ if for any $\epsilon>0, \delta>0$, there is a point $x \in \partial \Omega \cap B_{\delta}$ and $0<\mu<\delta$ $-\left|x^{0}-x\right|$ such that (5) holds with $B_{\delta}$ replaced by $B(x, \mu)$, for any $w$ satisfying $(6)$ in $B(x, \mu)$.

DEFinition 3. Let $\Omega \in H^{2, p}$. If for some positive $\epsilon_{0}, \delta$ and for any set $A \subset B_{\text {s }}$

(8) $\int_{\Lambda} L(\rho, w) d \lambda \geqq \epsilon_{0}\left\{\phi_{p}(A)+\psi_{p}(A)+(\text { vol. } A)^{1 / p}\right\}\left\{\int_{A}|w|^{2 q} d \lambda\right\}^{1 / q}$

for any $w \in C^{0}(A)$ satisfying (6) in $A$ and such that $1 \leqq|w(z)| \leqq 2$ in $A$, then we say that the strict $\mathrm{WLC}_{p}$ holds. The strict $\mathrm{WLC}_{p} I$ is defined similarly.

Note that if we omit the condition $1 \leqq|w| \leqq 2$ and replace $A$ by $V_{w}$, where $w \in C^{0}\left(B_{\delta}\right)$, then we obtain a condition which, although analogous to (5), is too restrictive if $p<\infty$, as seen by applying it to a sequence of $w$ 's whose support is $B_{\delta}$ but whose limit has its support in $B$, for any given $0<\eta<\delta$. Note also that all the results below remain unchanged if in (8) we replace (vol. $A)^{1 / p}\left\{\int|w|^{2 q} d \lambda\right\}^{1 / q}$ by $\int|w|^{2} d \lambda$. Finally, all the results remain true if we modify Definitions 1,2 and 3 by taking $w(x) \equiv$ const. such that (6) holds at one point of $B_{\delta}, B(x, \mu)$ and $A$ respectively.

\section{Statement of results.}

TheOREM 1. Let $\Omega \in H^{2, p}(1<p \leqq \infty)$. If $\rho \in H^{2, \infty}(N)$ where $N$ is some neighborhood of $x^{0}$, then (i) the $\mathrm{WLC}_{p}, \mathrm{WLC}_{p} I$ and the $\mathrm{LC}$ at $x^{0}$ 
coincide, and (ii) the strict $\mathrm{WLC}_{p}$, strict $\mathrm{WLC}_{p} I$ and the strict $\mathrm{LC}$ at $x^{0}$ coincide.

ThEOREM 2. Let $\Omega \in H^{2, p}(1<p \leqq \infty)$. If $\partial \Omega$ satisfies the strict $\mathrm{WLC}_{p}$ then $\Omega$ is a domain of holomorphy.

THEOREM 3. Let $\Omega \in H^{2, p}(1<p \leqq \infty)$. If $\Omega$ is a domain of holomorphy then $\partial \Omega$ satisfies the $\mathrm{WLC}_{p} I$.

Set $K=\bar{\Omega}$. Denote by $A(K)$ the algebra of uniform limits of functions holomorphic on $K$, by $S(K)$ the space of its maximal ideals, and by $\Gamma(K)$ its Shilov boundary. If $K$ is $S_{\delta}$, i.e., if $K=\bigcap_{m=1}^{\infty} \Omega_{m}$ where $\Omega_{m}$ are domains of holomorphy then (Rossi [4]) $S(K)=K$ and, if $\partial \Omega \in C^{2}, \Gamma(K)=\mathrm{Cl}(P(K))$ where $P(K)$ is the set of points of $\partial \Omega$ where the strict LC holds and "Cl" means "closure of." Furthermore, for each $\bar{x} \in P(K)$ there exists $f$ holomorphic on $K$ with

$$
|f(\bar{x})|>|f(z)| \quad \text { for all } z \in K, z \neq x \text {. }
$$

THEOREM 4. If $K$ is $S_{\delta}, \Omega \in H^{2, p}$ and $\partial \Omega$ satisfies the strict $\mathrm{WLC}_{p}$, then $\Gamma(K)=\partial \Omega$.

Theorem 4 can be extended to the case where the strict $\mathrm{WLC}_{p}$ holds only on a part (say $E$ ) of $\partial \Omega$, provided $\rho \in C^{2}$ in a neighborhood of $\partial \Omega \backslash \bar{E}$.

Finally, Theorems $2-4$ extend to domains $\Omega$ which are only locally given by functions $\rho$ in $H^{2, p}$. One uses a partition of unity in conjunction with the arguments given below.

5. Proofs outline. To prove $\mathrm{LC} \Rightarrow \mathrm{WLC}_{p}$, write

$$
\begin{aligned}
L(\rho(z), w(z))= & L\left(\rho(z)-\rho\left(x^{0}\right), w(z)\right)+L\left(\rho\left(x^{0}\right), w(z)-w^{0}\right) \\
& +L\left(\rho\left(x^{0}\right), w^{0}\right)
\end{aligned}
$$

and, given $\eta>0$, determine $\delta, w^{0}$ such that $\left|w(z)-w^{0}\right| \leqq \eta|w(z)|$ in $B_{\delta}$, and make use of (4) and of Hölder's inequality. To prove $\mathrm{WLC}_{p} I$ $\Rightarrow L C$, assume $L\left(\rho\left(x^{0},\right) w^{0}\right)<-\epsilon_{0}\left|w^{0}\right|^{2}$ and derive $L(\rho(z), w(z))$ $<-\epsilon_{0}\left|w^{0}\right| 2 / 2\left(w, w^{0}\right.$ related as above), then integrate. Since $W^{2} C_{p}$ $\Rightarrow \mathrm{WLC}_{p} I$, (i) follows. The proof of (ii) is similar.

To prove Theorem 2, take $\Omega_{m}=\left\{z ; z \in \Omega_{*}, \rho_{m}(z) \equiv \rho_{c_{m}}(z)-\delta_{m}<0\right\}$ with $\epsilon_{m} \rightarrow 0, \delta_{m} \rightarrow 0$, such that, $\Omega_{m} \supset \Omega_{m+1}$, and let $x^{0} \in \partial \Omega$ and $\epsilon_{0}, \delta$ as in Definition 3. Let $m \geqq m_{0}$ be such that $B_{\delta} \cap \partial \Omega_{m} \neq \varnothing$ and take $A \subset B_{\delta}$, $A \cap \partial \Omega_{m} \neq \varnothing$. Given $W \in C^{0}(A)$ satisfying (6) with $\rho$ replaced by $\rho_{m}$, choose $w \in C^{0}(A)$ satisfying (6), with $|W(z)-w(z)| \leqq \mu_{m}|W(z)|$, $\mu_{m} \rightarrow 0$ if $m \rightarrow \infty$. Writing 


$$
\begin{aligned}
\int_{A} L\left(\rho_{m}, W\right) d \lambda= & \int_{A} L(\rho, w) d \lambda+\int_{A} L\left(\rho_{m}-\rho, W\right) d \lambda \\
& +\int_{A} L(\rho, W-w) d \lambda
\end{aligned}
$$

and using Hölder's inequality and (8), derive (8) with $\rho, w, \epsilon_{0}$ replaced by $\rho_{m}, W, \epsilon_{0} / 2$ provided $m \geqq m_{1}$. Thus, the strict $\mathrm{WLC}_{p}$ (and, by Theorem 1, the LC) holds at each point $\bar{x} \in \partial \Omega_{m} \cap B_{\delta}$. By the HeineBorel Theorem, the LC then holds at all the points of $\partial \Omega_{m}$, for all large $m$; thus these $\Omega_{m}$ (and, necessarily, also $\Omega$ ) are domains of holomorphy.

To prove Theorem 3, let $x^{0} \in \partial \Omega$ and $\epsilon, \delta$ as in Definition 2. Choose $\phi \in C^{\infty}\left(C^{n}\right)$ satisfying: $\phi=1$ in $B\left(x^{0}, \delta / 2\right), \phi=0$ outside $B\left(x^{0}, \delta\right)$, $0 \leqq \phi \leqq 1$, elsewhere. Take $\tilde{\rho}_{\gamma}=\rho_{\gamma}-A_{\gamma}+B_{\gamma} \phi(z)$ with $A_{\gamma} \rightarrow 0, B_{\gamma} \rightarrow 0$ if $\gamma \rightarrow 0, B_{\gamma}>0$, such that the domain $\tilde{\Omega}$, defined by $\tilde{\rho}_{\gamma}<0$, is contained in $\Omega$ and $\partial \tilde{\Omega}$ touches $\partial \Omega$ at some point $\bar{x} \in B\left(x^{0}, \delta\right)$. Observing that not all the functions holomorphic in $\tilde{\Omega}$ can be continued into a neighborhood of $\bar{x}$, a result of Lewy [3] (see also [1, Chapter 2]) implies

$$
L\left(\tilde{\rho}_{\gamma}(\bar{x}), w\right) \geqq 0 \quad \text { whenever } \quad \sum \frac{\partial \tilde{\rho}_{\gamma}(\bar{x})}{\partial z_{j}} w_{j}=0 .
$$

Now use arguments as in the previous two proofs.

To prove Theorem 4, it suffices to show that for any $x^{0} \in \partial \Omega$ and $\delta>0$ there exists a point $\bar{x} \in \partial \Omega \cap B_{\delta}$ for which (9) holds. With $\phi$ as before, construct $\hat{\rho}_{\gamma}=\rho_{\gamma}-A_{\gamma}^{\prime}-B_{\gamma}^{\prime} \phi\left(A_{\gamma}^{\prime} \rightarrow 0, B_{\gamma}^{\prime} \rightarrow 0, B_{\gamma}^{\prime}>0\right)$ such that $\hat{\Omega}$, defined by $\hat{\rho}_{\gamma}<0$, contains $\Omega$ and $\partial \hat{\Omega}$ touches $\partial \Omega$ at some point $\bar{x}$ in $\partial \Omega \cap B_{\delta}$. Show that the result (9) of Rossi can be applied in $\hat{\Omega}$.

\section{REFERENCES}

1. L. Hörmander, Lectures on functions of several complex variables, Van Nostrand, Princeton, N. J., 1965 (to appear).

2. K. de Leeuw and $\mathrm{H}$. Mirkil, Majorations dans $L_{\infty}$ des operateurs différentiels a coefficients constants, C. R. Acad. Sci. Paris 254 (1962), 2286-2288.

3. $\mathrm{H}$. Lewy, On the local character of the solutions of an atypical linear differential equation in three variables and a related theorem for regular function of two complex variables, Ann. of Math. 64 (1956), 514-522.

4. H. Rossi, Holomorphically convex sets in several complex variables, Ann. of Math. 74 (1961), 470-493.

NORTHWESTERN UNIVERSITY 